\section{Free Communications}

\section{COACH AWARENESS AND PRACTICE IN RELATION TO GROWTH/MATURATION AND TRAINING LOAD IN YOUNG COMPETITIVE GYMNASTS: IMPLICATIONS FOR INJURY PREVENTION}

\footnotetext{
1,2Tejal Sarika Patel, ${ }^{2}$ Sean Cumming, ${ }^{2}$ Sean Williams, ${ }^{2}$ Bekker Sheree, ${ }^{1}$ Alex McGregor,
} ${ }^{1}$ Karen Williams, ${ }^{3}$ Louise Fawcett. 'British Gymnastics, Shropshire, UK; ${ }^{2}$ University of Bath, Department for Health, Bath, UK; ${ }^{3}$ British Gymnastics - English Institute of Sport, Sports Science and Sports Medicine Team, Shropshire, UK

10.1136/bjsports-2021-IOC.1

Background Growth/maturation and training load have been identified as risk factors for injury in youth sport. However, in gymnastics little is known regarding these risk factors for injury. Objective To understand current awareness and practice of coaches in relation to growth/maturation and training load in young, competitive British gymnasts.

Design Qualitative approach consisting of focus groups and inductive thematic analysis

Setting Men's artistic (MAG), Women's artistic (WAG) and Trampoline (TRA) coaches of Great Britain's National Development Programme (pathway) gymnasts.

Participants 44 (16 Female) pathway coaches took part in the study. 11 coaches worked within MAG, 15 within WAG and 18 within TRA gymnastics.

Assessment of Risk Factors Eight focus groups (2 MAG, 3 WAG, 3 TRA) were conducted to explore insights and experiences of coaches around the areas of growth/maturation and training load in relation to injury and its prevention. Data was analysed manually using an inductive thematic approach to identify core themes related to the study aim.

Main Outcome Measurements To determine the core themes and sub-themes related to growth/maturation and training load that were important to these coaches, specifically as related to injury and its prevention.

Results Coach awareness of the physical changes and injuries that occurred during growth/maturation was high across all disciplines. Psychological challenges and loss of skills were also identified as occurring during this time. Conversely, the awareness and practice of monitoring growth and maturity and training load for injury prevention varied between disciplines. Additionally, sports science and medicine practitioners were recognised as key stakeholders in managing and preventing injuries during growth.

Conclusions The study highlights coach awareness, experiences and challenges that accompanies growth and maturation in their gymnasts. This has implications and considerations for injury and its preventions, including early identification of the growth spurt, monitoring and modifying training in consultation with key stakeholders and further coach education.

002 ABSTRACT WITHDRAWN

\section{SURVIVAL ANALYSIS OF LOWER-LIMB APOPHYSEAL INJURIES IN YOUTH ELITE SOCCER IN ASSOCIATION WITH GROWTH AND SKELETAL MATURATION}

\begin{abstract}
${ }^{1,9}$ Olivier Materne, ${ }^{2}$ Abdulaziz Farooq, ${ }^{2}$ Karim Chamari, ${ }^{3}$ Amanda Johnson, ${ }^{2,7,8}$ Adam Weir, ${ }^{6}$ Per Hölmich, ${ }^{4}$ Matt Greig, ${ }^{4,5}$ Lars Mc Naughton. ${ }^{1}$ National Sports Medicine Program (NSMP), Aspetar, Doha, Qatar; ${ }^{2}$ Aspetar, Qatar Orthopaedic and Sports medicine Hospital, Doha, Qatar; ${ }^{3}$ Aspire Academy, Doha, Qatar; ${ }^{4}$ Edge Hill University Department of Sport and Physical Activity, Lancashire, UK; ${ }^{5}$ Department of Sport and Movement Studies, Faculty of Health Science, University of Johannesburg, Johannesburg, South Africa; ${ }^{6}$ Sports Orthopedic Research Center - Copenhagen (SORC-C), - Copenhagen University Hospital, Hvidovre, Denmark; ' Erasmus MC Center for Groin Injuries, Department of Orthopaedics, Erasmus MC University Medical Centre, Rotterdam, Netherlands; ${ }^{8}$ Sport medicine and exercise clinic Haarlem (SBK), Haarlem, Netherlands; ${ }^{9}$ Qatar Football Association, Doha, Qatar
\end{abstract}

\subsection{6/bjsports-2021-IOC.2}

Background Adolescent athletes have different injury patterns to those of adults, and their growing bones are susceptible to overuse injuries. Evidence of growth-related injuries in relation to growth and maturation in youth elite soccer players remains sparse.

Objective 1) to investigate lower-limb apophyseal injuries (LL$\mathrm{AI}$ ) and 2) their relationship with peak height velocity (PHV) and skeletal age (SA) in elite young soccer players.

Design Prospective longitudinal cohort study.

Setting Youth elite soccer academy.

Patients (or Participants) During 4 consecutive seasons, 551 youth soccer players from under U-9 (under 9yrs.) to U-19 with a mean age of $14.9 \pm 1.7$ years participated.

Interventions (or Assessment of Risk Factors) Injury surveillance Main Outcome Measurements Lower-limb apophysis items were integrated in the consensus injury registration form. Diagnoses were established by experienced clinicians and the team's dedicated physiotherapist. Imaging was utilized if required.

Skeletal age was assessed at the start of each season (Fels method), then classified by maturity status (SA minus chronological age: Late: >-1 yr; Normal: +/-1 yr; Early: <1 yr \& SA $<18$ yr; Mature: $\mathrm{SA}=18 \mathrm{yr}$ ).

Peak height velocity was calculated non-invasively (Mirwald method) three times during each season, then allocated to Pre-circum <-1 yr from PHV), Circum- ( $\geq-1$ yr from/to PHV $\leq+1$ ) or Post-circum ( $>1$ yr to PHV) status.

Results There were 307 (14\% of 2204) growth-related injuries recorded, of which $84 \%$ were LL-AI. After controlling for confounders, adjusted injury-free survival analysis showed substantially different hazard ratios (HR) for LL-AI (i) PHV: Circum higher than Pre-circum (HR: 2.09, 95\% CI, 1.22-3.56; $\mathrm{p}<0.05$ ) and Post-circum (HR: 1.88, 95\% CI, $1.22-2.90 ; \mathrm{p}<0.05)$ respectively. (ii) SA: Mature lower than Late-maturers $(\mathrm{HR}=2.56, \quad 95 \% \quad \mathrm{CI}$ : $1.01-6.51$; $\mathrm{p}<0.05)$, Normal-maturers $(\mathrm{HR}=2.14,95 \% \mathrm{CI}: 1.30-3.50$; $\mathrm{p}<0.05)$ and Early-maturers $(\mathrm{HR}=1.82$, 95\% CI: $1.11-$ $2.97 ; \mathrm{p}<0.05)$ respectively.

Conclusions Apophyseal injuries of the lower-limb is the most prevalent growth-related injury in youth soccer. The growth spurt was the period of greatest risk for apophyseal injury. However, the type and location of apophyseal injuries varies depending on growth and skeletal maturation status. 